\title{
Eco-environmental Assessment and Analysis of Tonglvshan Mining area in Daye City, Hubei Province Based on Spatiotemporal methodology
}

\author{
X.M.Zhang ${ }^{\mathrm{a}, \mathrm{b}}$, G.J.He, ${ }^{\mathrm{a}, *}$, M.M. Wang ${ }^{\mathrm{a}, \mathrm{b}}$, Z.M. Zhang ${ }^{\mathrm{a}}$,W.L. Jiao ${ }^{\mathrm{a}}$,Y.Peng ${ }^{\mathrm{a}}$,G.Z. Wang ${ }^{\mathrm{a}}$,H.C. Liu ${ }^{\mathrm{a}, \mathrm{b}}$, T.F. Long ${ }^{\mathrm{a}, \mathrm{b}}$ \\ ${ }^{a}$ Institute of Remote Sensing and Digital Earth, Chinese Academy of Sciences, Beijing 100094,China- \\ (zhangxm, hegj,wangmm,zhangzm,jiaowl,pengy,wanggz,liuhc,longtf)@ radi.ac.cn \\ ${ }^{b}$ University of Chinese Academy of Sciences, Beijing 100049, China
}

KEY WORDS: Spatiotemporal Principles/Patterns, Eco-environmental Assessment, Eco-environmental Quality Index (EQI), Land Cover/Use, Buffers, Township Administrative Units, Daye City

\begin{abstract}
:
Mine exploitation has a significant impact on the ecological environment status of the surroundings. To analyze the impact of Tonglvshan Mining area to its surroundings, this paper adopted the spatiotemporal methodology based on the extracted Ecoenvironmental Quality Index (EQI) to analysis the extent and degree of the effect. The spatiotemporal methodologies are based on two scales: buffers and administrative units. EQI includes Biological Abundance Index (BAI), Vegetation Index (VI), Water Network Density Index (WNDI), and Land Degradation Index (LDI). The weight of each Index was determined by the analytic hierarchy process (AHP) and scores of the experts. The calculating of EQI was referenced to the standard "Technical criterion for Eco-environment Status Evaluation" (HJ/T192-2006) and the "Standards for Classification and Gradation of Soil Erosion" (SL 190-96) . Considering ecological and environmental characteristics relevant to China, this method has been widely used to study the environment status of specific regions in China. The assessment based on buffers adopted the radius of $300 \mathrm{~m}, 500 \mathrm{~m}, 700 \mathrm{~m}, 1000 \mathrm{~m}$, $1500 \mathrm{~m}, 2000 \mathrm{~m}, 2500 \mathrm{~m}, 3000 \mathrm{~m}, 3500 \mathrm{~m}$, and $4000 \mathrm{~m}$ as the buffers in 3 typical miners respectively. The calculated result indicates that, the REI is increasing with the radius and the increasing rate becoming smaller until REI is stable. Which means the effect of miner is getting weaker with the distance to the miner is increasing and the effect is diminished when the distance is far enough. The analysis of the 3 typical miner shows that the extent and degree of the effect of miner relates not only with the area of the miner, but also with type of mineral resource, the status of mining and the ecological restoration. The assessment was also carried out by calculating the EQI in 14 administrative units in Daye city in 2000, 2005, and 2010. The study shows that the EQI is decreasing in 14 units from 2000 to 2010 . The spatiotemporal analysis of the type and area of land cover in 14 units within ten years period ranging from 2000 to 2010 shows that the mainly factor to affect the eco-environment status is mine exploitation and urban expansion.
\end{abstract}

\section{INTRODUCTION}

Ecosystems and ecosystem services are essential for human survival and sustainable development. Ecosystem assessment is the bridge to link the findings of ecology and natural sciences with economy and political decision-making(Ouyang et al., 2014). Irrational exploitation and utility of resources and extensive economic growth have become the primary causes of deterioration of ecological environment in many places in China(Dai et al., 2012). The disturbance from human activities has generated great pressure on the regional eco-environment resulting in a reduction in biodiversity, a decrease in water and air quality and life quality of local inhabitants and an increasing of natural disasters (Herrera-Silveira and Morales-Ojeda, 2009; Li et al., 2010; Zhou and Wang, 2014). Such impacts are an urgent call for ecological zoning and planning with various techniques and methods in order to regulate human activities and guide the sustainable use of resources and ecological services (Ignatieva et al. 2011; Qinet al. 2011). On this background, we need to monitor the eco-environmental status and trends by developing methods and theoretical support for environmental planning and management. Daye has been selected as one of the five typical mineral resource mining area in china, which is a sub-task of National Ecosystem Survey and Assessment of China $(2000-2010)$. This project was approved by State Council of China, jointly launched by Ministry of Environmental Protection and Chinese Academy of Sciences. The purpose of this task is to discover the ecosystem status and ecological problems and their change trends in China.

The purpose of this study is to provide a method for assessing the ecological status in Daye, a typical mining city in China. In order to analyze the impact of Mining area to its surroundings, we adopted two methods which are based on buffers and township administrative units. Using extracted Land cover/use type, Remote Sensing data and DEM as the data source, applying EQI model as evaluation criterion, we calculate and analysis a series of the values for buffers and township administrative units. The result of this study is intended to serve as a technical support and decision-making for the relevant department to set up plans for mineral resource exploitation, and to cater the need of harmonious development of environment and economy.

\section{STUDY AREA AND DATA SOURCES}

\subsection{Study Area}

Our study area encompasses the entire territory of Daye, covering $1593 \mathrm{~km} 2$. The Daye city region $\left(29.67^{\circ}-30.25^{\circ} \mathrm{N}\right.$,

\footnotetext{
* Corresponding author
} 
$\left.114.52^{\circ}-115.35^{\circ} \mathrm{E}\right)$ situates to south of the Yangtze River, the southeast of Hubei province (Fig.1).

As the birthplace of World Bronze, Daye is an industrial city, a center of mining and metallurgy in China. Its name Daye means Big Smeltery. Daye is known for copper-containing minerals in history, Now, Known for its iron ore mining and processing. TonglVshan Mine is located just southwest of the modern city.

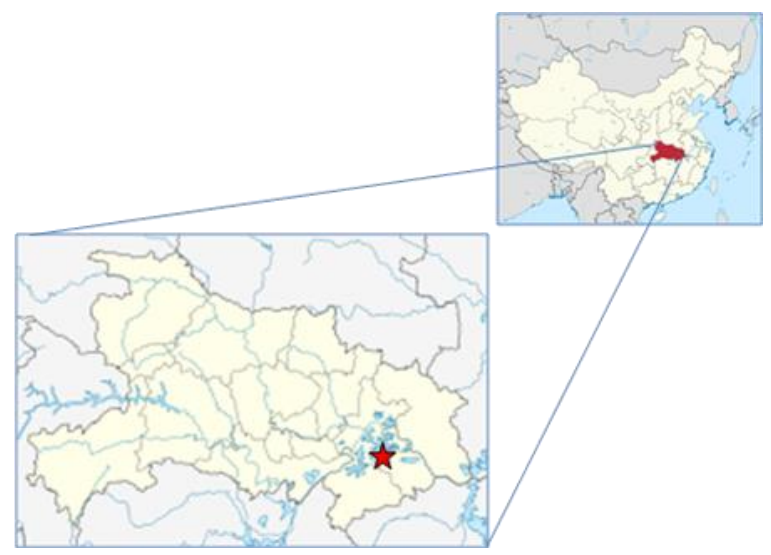

Fig.1 Location map of the study area

\subsection{Data and Processing}

This paper collects research data in two kinds, Remote Sensing data and GIS data. Remote Sensing data is mainly SPOT images, as well as ALOS and Landsat, which is at a five-year interval from 2000 to 2010. The GIS data is mainly administrative boundary and DEM (Table.1).

\begin{tabular}{|l|l|l|l|}
\hline ID & Satellite & $\begin{array}{l}\text { resolutio } \\
\mathrm{n}\end{array}$ & $\begin{array}{l}\text { Acquired } \\
\text { date }\end{array}$ \\
\hline 1 & SPOT-2 & $10 \mathrm{~m}$ & 20000614 \\
2 & Landsat-ETM+ & $30 \mathrm{~m}$ & 20001101 \\
3 & SPOT-2 & $10 \mathrm{~m}$ & 19990803 \\
4 & SPOT-5 & $2.5 \mathrm{~m}$ & 20050929 \\
5 & SPOT-5 & $2.5 \mathrm{~m}$ & 20050929 \\
6 & ALOS Multi-spectral & $10 \mathrm{~m}$ & 20101106 \\
7 & ALOS Multi-spectral & $10 \mathrm{~m}$ & 20101205 \\
8 & ALOS Pan & $2.5 \mathrm{~m}$ & 20101106 \\
9 & ALOS Pan & $2.5 \mathrm{~m}$ & 20101106 \\
10 & ALOS Pan & $2.5 \mathrm{~m}$ & 20101106 \\
11 & ALOS Pan & $2.5 \mathrm{~m}$ & 20101205 \\
\hline
\end{tabular}

Table1. Remote Sensing data source

\begin{tabular}{|l|l|l|}
\hline Name & Format & $\begin{array}{l}\text { resolution/scal } \\
\mathrm{e}\end{array}$ \\
\hline $\begin{array}{l}\text { Administrative } \\
\text { boundary }\end{array}$ & vector & Township level \\
ASTER DEM & grid & $30 \mathrm{~m}$ \\
\hline
\end{tabular}

Table2. GIS data source

The image processing included ortho-rectation, fusion, mosaicing and clipping according to administrative boundary. The result of the processing is shown in upper Fig.2. Based on the images in Daye in 2000, 2005, and 2010, we selected the Object-oriented classification and Back Tracing classification to extract the land cover/use types (Fig.2 lower). Object-oriented classification is suitable for high-resolution image classification and Back-tracing method works better in compare with the classification result in different time.

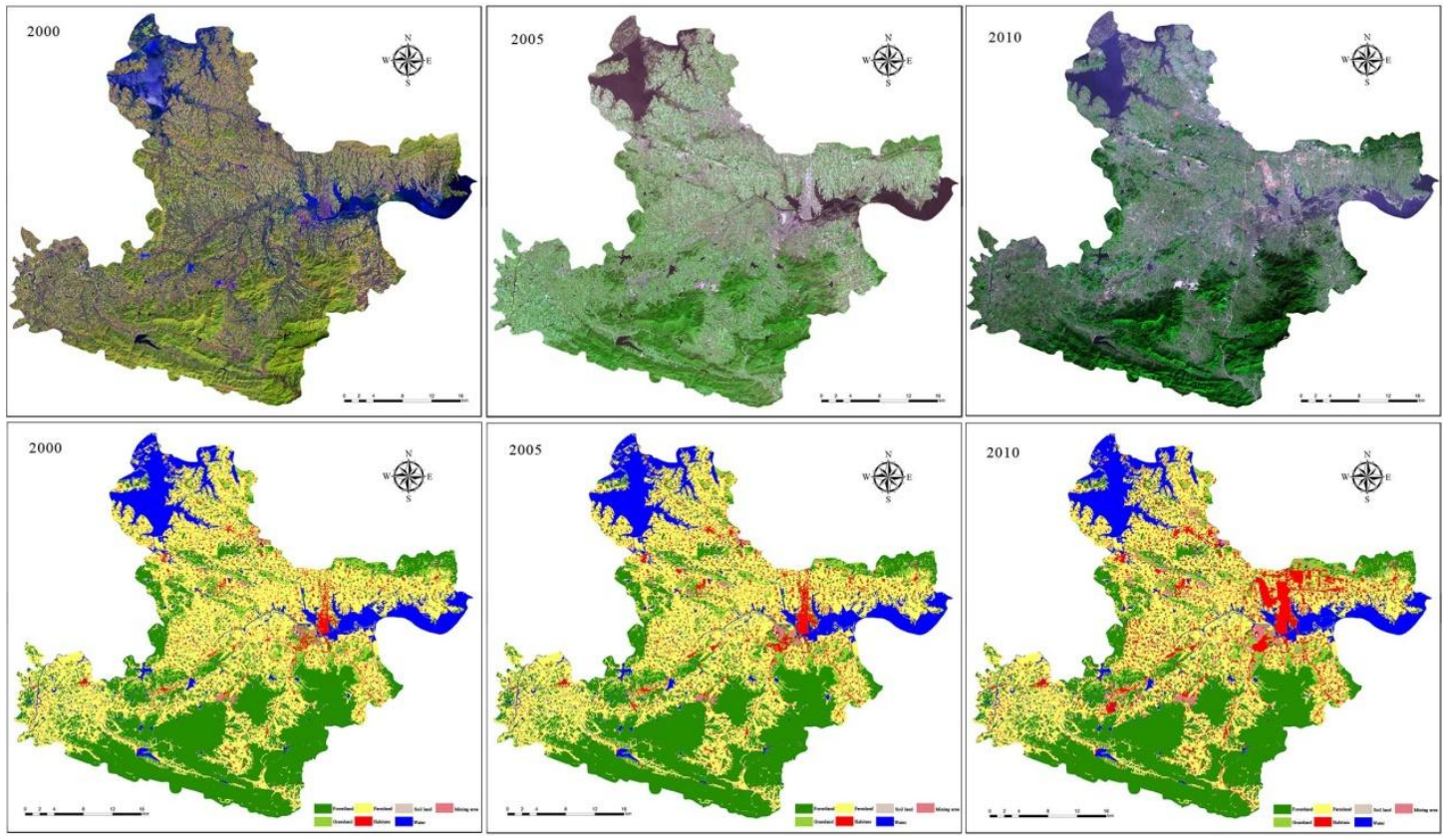

Fig2. Image and classification of Daye in 2000, 2005, and 2010

\section{METHODOLOGY}

There are many methods to evaluate regional environmental quality, including principal component analysis, set pair analysis (Chen et al., 2009), fuzzy evaluation (Fu et al., 2005), analytic hierarchy process (AHP) (Li, 2009), and index evaluation ( $\mathrm{Li}, 2009)$. Eco-environment quality index is a useful tool for environment assessment. With the recent development 
of geospatial technologies, such as remote sensing and geographic information systems (GIS), its assessment procedures can be significantly enhanced (Zhang et al., 2003; Li et al., 2005).

The State Environmental Protection Administration of China (SEPA) published its "Technical Criterion for Ecoenvironmental Status Evaluation" in 2006. This established an Eco-environmental Quality Index (EQI) model to evaluate environment status in China via Biological Abundance Index (BAI), Vegetation Index (VI), Water Network Density Index (WNDI), Land Degradation Index (LDI) and Pollution Index (PI). Considering ecological and environmental characteristics relevant to China, this method has been widely used to study the environment status of specific regions in China. But with the difficult to acquire the statistical datum of Pollution in township level, this paper selected four indicators by which to evaluate ecological and environmental problems and status in Daye.

Due to differences in parameter dimensions and magnitudes, the quantitative indexes are first normalized prior to calculation, as shown in Equation (1):

$X=\frac{X_{i}-X_{\min }}{X_{\max }-X_{\min }} * 100$

Here, $\mathrm{X}$ is the result after normalization, $\mathrm{Xmax}$ is the maximum value and $\mathrm{Xmin}$ is the minimum value.

After calculating the four indexes above, the final evaluation model is designed using the comprehensive index method as (2):

$\mathrm{EQI}=\sum_{\mathrm{i}=1}^{4} \mathrm{X}_{\mathrm{i}} * \mathrm{~W}_{\mathrm{i}}$

Here, EQI denotes the Eco-environmental Quality Index, $\mathrm{Xi}$ is the ith index and Wi is the weight of the ithe index.

In this study, we use AHP analysis to define index weight and invite experts as decision makers. The weight of each index can be seen in Table3:

Tab3. The weight of each index in EQI

\begin{tabular}{lllll}
\hline Index & BAI & VI & WNDI & LDI \\
\hline Weight & 0.34 & 0.26 & 0.1 & 0.3 \\
\hline
\end{tabular}

\subsection{Assessment Based on Buffers}

The assessment based on buffers adopted the radius of $300 \mathrm{~m}$, $500 \mathrm{~m}, 700 \mathrm{~m}, 1000 \mathrm{~m}, 1500 \mathrm{~m}, 2000 \mathrm{~m}, 2500 \mathrm{~m}, 3000 \mathrm{~m}, 3500 \mathrm{~m}$, and $4000 \mathrm{~m}$ as the buffers in 3 typical miners respectively marked with Ore1, Ore2 and Ore3(Fig.3). Ore1, covering $0.5686 \mathrm{~km} 2$, width 800 metre, longth 1500 metre, locating on the edge of Baoan town and Jinshan town, which are all on the hills; Ore 2 including the $2.024 \mathrm{~km} 2$ mining field and $1.0013 \mathrm{~km} 2$ tailings, is near to urban zone, and is the oldest mine; Ore3 locating in Chengui town, has an area of $1.4841 \mathrm{~km} 2$ mining field and $0.9003 \mathrm{~km} 2$ tailings and located on hills.

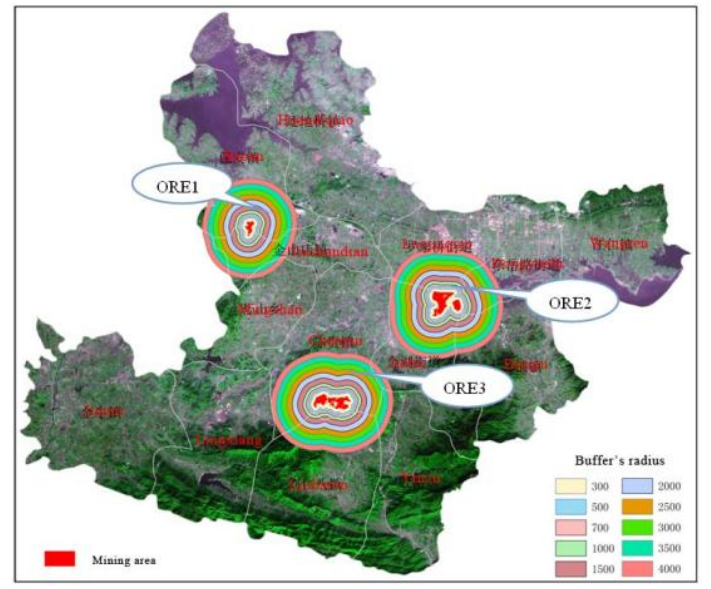

Fig3. The distribution of the 3 typical miners with buffers

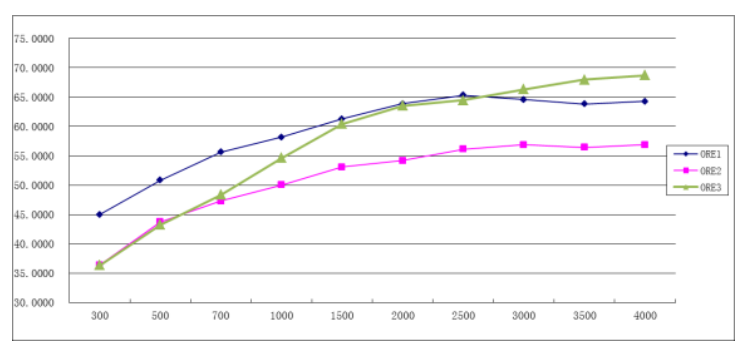

Fig.4 Calculate the EQI of all the buffers

The calculated result indicates that, the EQI is increasing with the radius and this show the eco-environment gets better with the effect of miner is getting weaker; meanwhile, the increasing rate becoming smaller until EQI tends to becomes stable. Which means the effect of miner is getting weaker with the distance to the miner is increasing and the effect is diminished when the distance is far enough. So we can use the value of EQI to mark the effect of miner to its surroundings, and the region of stable value to determine the effective region.

When the radius is less than 1500 meter, the EQI of Ore 1 is bigger than Ore2 and Ore3, which show that the ecoenvironment surrounded Ore1 is better than the other two miners'. Its reason lies in the area of miner, for the area of Ore 1 is $1 / 6$ of Ore 2 and $1 / 5$ of Ore 3 ; when the radius is greater than 1500 meter, the EQI of Ore 1 and Ore 3 are obviously great than Ore2, the possible reason is the location of the miner, for the Ore2 is situated to urban zone while the other two miners are on hills. The forest on the hill reduces the effect of miner to its surroundings.

The analysis of the 3 typical miner shows that the extent and degree of the effect of miner relates not only with the area of the miner, but also with type of mineral resource and its surroundings.

When the radius is great than or equal to 2000 meter, the EQI of Ore1 reaches a stable value of 64 (Variation less than 2). It shows the effective region of Ore 1 is 2000 meter. When the radius is great than 2500 meter, the EQI of Ore2 reaches a stable value of 56(Variation less than 2). So the effective region of Ore 2 is 2500 meter. And so on with Ore3, its effective region is 3000 meter reaching a stable value of 66 (Variation less than 2).

In addition, the intensity and region of miner to its surroundings is not only with the area, but also to the type of mineral resource 
and status of mining, eco-environmental restoration. With limits to the information from Remote Sensing image, this paper qualitative figures out the intensity and region of miner to its surroundings.

\subsection{Assessment Based on Administrative Units}

The assessment was also carried out by calculating the EQI in 3 subdistricts 10 towns and 1 township total 14 administrative units in 2000, 2005, and 2010. These administrative units are Dong yuelu subdistict, Jinhu subdistrict, Luojiaqiao subdistrict, Jinniu town, Baoan town, Lingxiang town, Jinshandian town, Huandiqiao town, Yinzu town, Wangren town, Liurenba town, Chengui town, Dajipu town and Mingshan township.

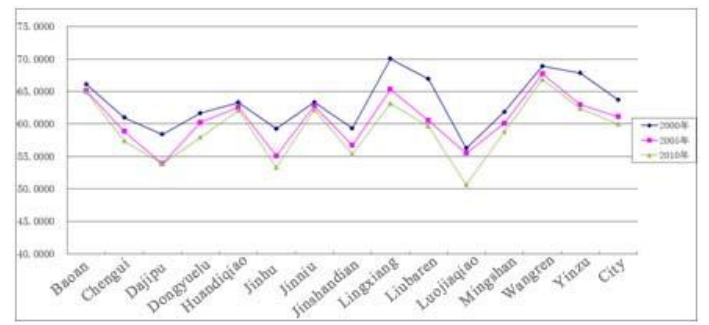

Fig.5 EQI of 14 administrative units in Daye

The curves in Fig.5 show that, the EQI of 14 administrative units in Daye is decreasing as a whole from 2000 to 2010. As to statistical data, the variable quantity of EQI in Baoan town, Huandiqiao town and Jinniu town are all less than 2, which indicates there is no significant change of eco-environment in those 3 towns; The EQI in Chengui town, Dajipu town, Dongyuelu subdistict, Jinshandian town, Mingshan township, Wangren town and Yinzu town are all tend to decreasing with a range from 2 to 5 , which indicates there is a slightly deterioration of eco-environment in those 7 towns; however, the EQI in Dongyuelu subdistrict, Jinhu subdistrict, Lingxiang town and Luojiaqiao subdistrict are distinctly declining with a range from 5 to 10 , which indicates the eco-environment in those 4 town are severely deteriorated.

The oldest and greatest TongLvShan miner is located in Jinhu subdistict, which is the possible cause resulted in the worst ecoenvironment in all the 14 units. Moreover, the area of town region in Jinhu town is expanding from $6.5 \mathrm{~km} 2$ to $11 \mathrm{~km} 2$, the extensive urban expansion is also resulted in the worst ecoenvionment in Jinhu town. The area of mining in Lingxiang town is increasing from $0.52 \mathrm{~km} 2$ to $1.91 \mathrm{~km} 2$, those rapid increasing of mining region deteriorate the eco-environment in Lingxiang town. For Luojiaqiao subdistrict and Dongyuelu subdistrict, there are lare-scale construction of economic development zone, which result in the enormous increasing of building area.

In consequence, the spatiotemporal analysis of the type and area of land cover in 14 administrative units from 2000 to 2010 shows that the mainly factor affecting the eco-environment status is mine exploitation and urban expansion.

\section{CONCLUSION AND DISCUSSION}

This study examined eco-environmental status in two spatial scales via EQI calculated from remote sensing data. It modelled the Eco-environmental Quality Index (EQI) by extracting the four Index, BAI, VI, WNDI, and LDI, and evaluated and compared the environment status of Daye in 2000, 2005 and
2010. From the findings, the following points can be summarized: (1) In general, ecological environmental status in Daye is declining from 2000 to 2005, and to 2010. (2) The intensity and region of the effect of miner to its surroundings is not only with the area, but also to the type of mineral resource and status of mining. (3) The mainly factor affecting the ecoenvironment status in Daye is mine exploitation and urban expansion.

With limits to the information from Remote Sensing image, this paper qualitative figures out the intensity and region of the effect of miner to its surroundings. Besides, we discussed four factors affecting environmental quality in China, but in practice EQI is influenced by many factors and the analysis in this article are therefore incomplete. Future studies will discuss additional factors such as energy consumption, and will also examine these factors in combination.

\section{ACKNOWLEDGEMENTS}

This research was funded by the National Natural Science Foundation of China (No. 61401461) and National Ecological Environment Change Assessment by Remote Sensing Survey Project 2000-2010 (STSN-10-03).

\section{REFERENCES}

Chen J, Wang W S, Chen Y, 2009. Study on nationwide ecological environment level evaluation based on set pair analysis. Water Resources and Power, 27(2), pp.40-43.

Dai, X., Li, Z., Lin, S., Xu, W., 2012. Assessment and zoning of eco-environmental sensitivity for a typical developing province in China. Stochastic Environmental Research and Risk Assessment 26, pp.1095-1107.

Fu Z, Zhou Y X, Liu D W et al., 2005. Research on spatial fuzzy comprehensive assessment of eco-environmental quality: A case study in the east of Jilin Province. Journal of Arid Land Resources and Environment, 19(5), pp.98-101.

Herrera-Silveira, J.A., Morales-Ojeda, S.M., 2009. Evaluation of the health status of a coastal ecosystem in southeast Mexico: Assessment of water quality, phytoplankton and submerged aquatic vegetation. Marine Pollution Bulletin, Assessing Ecological Integrity in Marine Waters, using Multiple Indices and Ecosystem Components 59, pp. 72-86.

Li K, 2009. Application of analytical hierarchy process to integrate evaluation of eco-environment. Environmental Science \& Technology, 32(2) , pp. 183-185.

Li, Y., Zhu, X., Sun, X., Wang, F., 2010. Landscape effects of environmental impact on bay-area wetlands under rapid urban expansion and development policy: A case study of Lianyungang, China. Landscape and Urban Planning 94, pp. 218-227.

Ministry of water resources of the People's Republic of China. 1997. Standards for classification and gradation of soil erosion (SL 190-96), Chinese Water Power Press, Beijing, China.

Ouyang, Z., Wang, Q., Zheng, H., Zhang, F., Hou, P., 2014. National Ecosystem Survey and assessment of China (2000 - 
2010). Bulletin of Chinese Academy of Sciences 29, pp. 462 466.

State Environmental Protection Administration. 2006. Technical criterion for eco-environmental status evaluation, (HJ/T192-2006), China Environment Science Press, China.

Zhou, J., Wang, L., 2014. Comprehensive study on ecological restoration and land exploitation of mining subsidence in suburbs of Chinese mining cities - Springer. International Journal of Coal Science \& Technology 1, pp. 248-252. 